\title{
Pseudolithiasis due to Ceftriaxone Treatment for Meningitis in Children: Report of 8 Cases
}

\author{
Nilgun Araz, ${ }^{1}$ Vahap Okan, ${ }^{3}$ Mustafa Demirci ${ }^{2}$ and Mustafa Araz ${ }^{3}$ \\ ${ }^{1}$ Department of Pediatrics, Child Hospital, Gaziantep, Turkey \\ ${ }^{2}$ Department of Radiology, Child Hospital, Gaziantep, Turkey \\ ${ }^{3}$ Department of Internal Medicine, Gaziantep University, Medical Faculty, Gaziantep, Turkey
}

Araz, N., Okan, V., Demirci, M. and Araz, M. Pseudolithiasis due to Ceftriaxone Treatment for Meningitis in Children: Report of 8 Cases. Tohoku J. Exp. Med., 2007, 211 (3), 285-290 — Cholelithiasis rarely occurs in childhood. Ceftriaxone is a widely used antimicrobial agent in pediatrics due to the broad spectrum. Reversible biliary sludge and/or lithiasis, named as pseudolithiasis, have been reported in patients treated with ceftriaxone. We observed ceftriaxone-associated pseudolithiasis in 8 patients with meningitis. The aim of this study was to report the clinical characteristics of these patients and to evaluate the related factors for the development of ceftriaxone-associated pseudolithiasis in children. The study group consisted of 7 boys and 1 girl. All patients received ceftriaxone $100 \mathrm{mg} / \mathrm{kg} / \mathrm{day}$ for meningitis. The ultrasonographic evaluation was performed on 5th-10th days after the initiation of the therapy. Biliary sludge was detected in one patient, and gallstone was detected in three patients, while biliary sludge with gallstone was detected in four patients. Six of the cases were diagnosed during summer time. Thus, high temperature may cause loss of fluid, leading to easier formation of sludge. Ceftriaxone treatment was discontinued after sonographic demonstration of pseudolithiasis. Gallbladder sonograms were found to be normal in all patients at the follow-up sonographic examinations performed after 30 days of the diagnosis without specific treatment. Clinicians should screen all pediatric patients living in areas with high temperature and receiving ceftriaxone treatment (over $100 \mathrm{mg} / \mathrm{kg}$ ) by ultrasonography for biliary sludge or gallstone formation even if they are asymptomatic. —— pseudolithiasis; biliary sludge; ceftriaxone; children

(C) 2007 Tohoku University Medical Press

During childhood, cholelithiasis is a rare problem and it is usually associated with hemolytic disorders, congenital abnormalities of bile ducts, total parenteral nutrition, diseases of the terminal ileum and bone marrow or solid organ transplantation (Ko et al. 1999). Cholelithiasis may also develop due to use of some medications.
Ceftriaxone and octreotide are the most common drugs for drug-associated biliary sludge (Ko et al. 1999). Ceftriaxone is a semi-synthetic parenteral third generation cephalosporin and a widely used antimicrobial agent in pediatric practice due to broad spectrum, long plasma half-life and relatively low adverse effects. Reversible biliary

Received December 8, 2006; revision accepted for publication January 22, 2007.

Correspondence: Nilgun Araz, M.D., Gaziantep University, Medical Faculty, Department of Pediatrics, 27310

Gaziantep, Turkey.

e-mail: naraz@gantep.edu.tr 
sludge and/or lithiasis, named as pseudolithiasis, have been reported in patients treated with ceftriaxone (Lopez et al. 1991). Pseudolithiasis due to ceftriaxone can occur both in children and adults. In clinical practice, we observed ceftriaxoneassociated pseudolithiasis in 8 patients with meningitis. The aim of this study was to report the clinical characteristics of these patients and evaluate the related factors for the development of pseudolithiasis in children.

\section{Patients}

The patients were followed between July 2005 and March 2006 at the Child Hospital of Gaziantep. The study protocol was not arranged as a prospective study. We observed ceftriaxoneassociated pseudolithiasis in one patient with meningitis. Thereafter, we followed children receiving ceftriaxone for meningitis and we found out other 7 cases with pseudolithiasis. The patient group consisted of 7 boys and 1 girl. Mean age of the children was $6 \pm 2$ years ( 3 to 9 years). Clinical and demographic characteristics of the children are summarized in Table 1 . None of the patients had hemolytic anemia, renal or liver failure, ileal disease, cystic fibrosis, malignancy or received total parenteral nutrition. None of them were taking other drugs known to be associated with biliary sludge and/or lithiasis.

Hematological (hemoglobin, white blood cell count, platelet count) and biochemical analysis of the blood (total and direct bilirubin, liver function tests, blood urea nitrogen, creatinine and lipids) were performed in all subjects. Biochemical parameters were within normal limits except for one patient who had an elevated serum alkaline phosphatase level (1.5 fold of the upper limit), but serum bilirubin levels were normal for this subject.

All patients received ceftriaxone therapy 100 $\mathrm{mg} / \mathrm{kg}$, given by bolus intravenous injection once a day for meningitis. Two patients also received sultamycilin $100-200 \mathrm{mg} / \mathrm{kg} /$ day and two patients were given penicillin-G $100.000 \mathrm{U} / \mathrm{kg} /$ day in combination with ceftriaxone.

The ultrasonographic evaluation was ordered routinely by the pediatrician during the hospital stay and performed at $7 \pm 1$ days (5-10 days) of ceftriaxone treatment by the same radiologist using Sonoline Adara (Siemens, Erlangen, Germany) with a $3.5 \mathrm{MHz}$ convex or $5 \mathrm{MHz}$ linear array transducer. Sonographic examinations of gallbladder, intra- and extra-hepatic bile ducts, liver, spleen and kidneys were done. Abnormal gallbladder sonograms were defined as gallstone and biliary sludge. Biliary sludge was diagnosed by low-amplitude echoes within the lumen of gallbladder with no post-acustic shadows and gallstone was diagnosed by mobile hyperechogenic material with post-acustic shadows. Biliary sludge was detected in one patient, and gallstones were detected in three

TABLE 1. Clinical and demographic characteristics of the patients.

\begin{tabular}{cccccccrc}
\hline Case & $\begin{array}{c}\text { Age } \\
(\text { years })\end{array}$ & Sex & On admission & $\begin{array}{c}\text { Weight } \\
(\mathrm{kg})\end{array}$ & $\begin{array}{c}\text { B.tem } \\
\left({ }^{\circ} \mathrm{C}\right)\end{array}$ & $\begin{array}{c}\text { BP } \\
(\mathrm{mmHg})\end{array}$ & $\begin{array}{c}\text { HR } \\
(/ \mathrm{min})\end{array}$ & U density \\
\hline 1 & 7 & M & July 2005 & 15 & 40 & $110 / 70$ & 120 & 1,030 \\
2 & 6 & M & Aug 2005 & 17 & 38 & $110 / 70$ & 90 & 1,020 \\
3 & 5 & M & Aug 2005 & 30 & 38 & $110 / 65$ & 110 & 1,020 \\
4 & 5 & M & Aug 2005 & 30 & 38 & $100 / 65$ & 90 & 1,020 \\
5 & 6 & M & Aug 2005 & 17 & 37.5 & $115 / 75$ & 100 & 1,015 \\
6 & 3 & M & Sep 2005 & 15 & 38.5 & $105 / 60$ & 120 & 1,025 \\
7 & 5 & F & Oct 2005 & 15 & 40 & $105 / 65$ & 120 & 1,030 \\
8 & 9 & M & Mar 2006 & 16 & 37 & $80 / 50$ & 100 & 1,015 \\
\hline
\end{tabular}

M, male; F, female; Aug, August; Sep, September; Oct, October; Mar, March; B.tem, body temperature; $\mathrm{BP}$, blood pressure; HR, heart rate; $\mathrm{U}$, urine. 
patients (with a longest diameter of $1.5 \mathrm{~cm}$ ), while biliary sludge with gallstone was detected in four patients. Gallstone in the latter patients was in milimetric sizes in three patients and it was $0.5 \times$ $0.5 \mathrm{~cm}$ in one patient. Sonographic appearance of biliary sludge, gallstone and biliary sludge with gallstone is shown in Figs 1, 2, 3, respectively. Nephrolithiasis was not detected in any patient.

Ceftriaxone treatment was withdrawn in all patients after the diagnosis of pseudolithiasis.
Patients were scheduled for follow-up ultrasonography 30 days after the diagnosis of pseudolithiasis, however repeat ultrasonographic examination was performed $41 \pm 23$ days after discontinuation of ceftriaxone treatment due to some late referrals. Gallbladder sonograms were found to be normal in all patients at the follow-up sonographic examinations. The timing and results of the sonographic examinations of each patient are shown in Table 2.

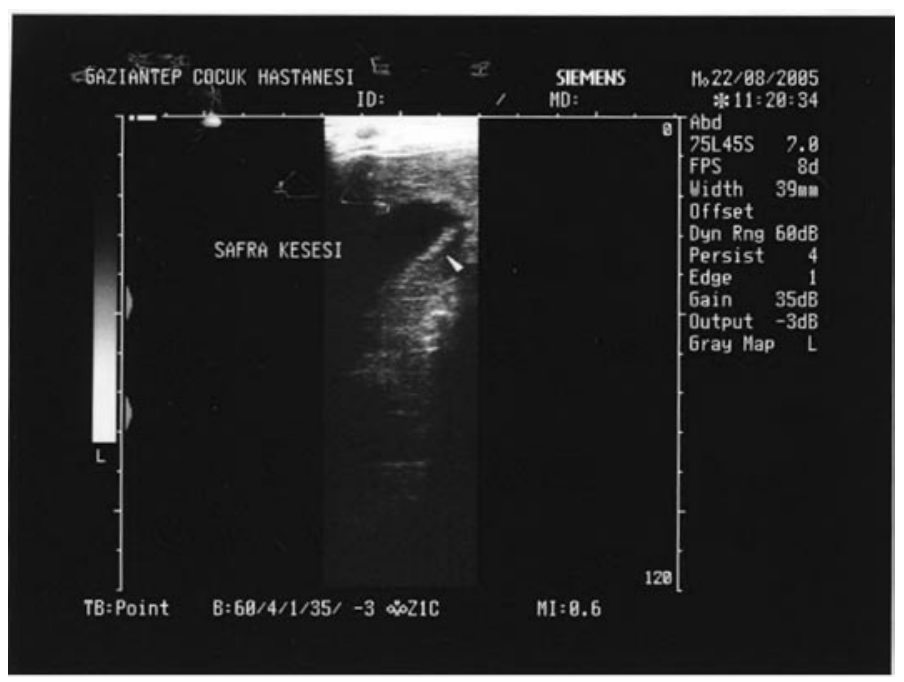

Fig. 1. Ultrasonographic image of biliary sludge.

Arrowhead showing low-echogenic particles without post-acustic shadows indicating sludge in the gallbladder lumen.

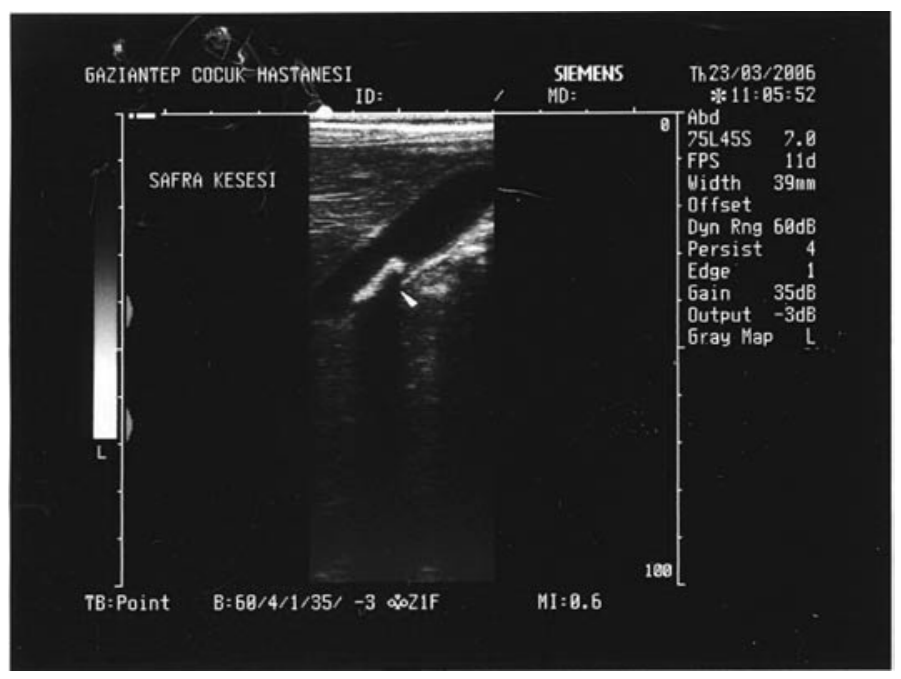

Fig. 2. Ultrasonographic image of gallstone.

Arrowhead pointing to the gallstone with accompanying post-acustic shadows. 


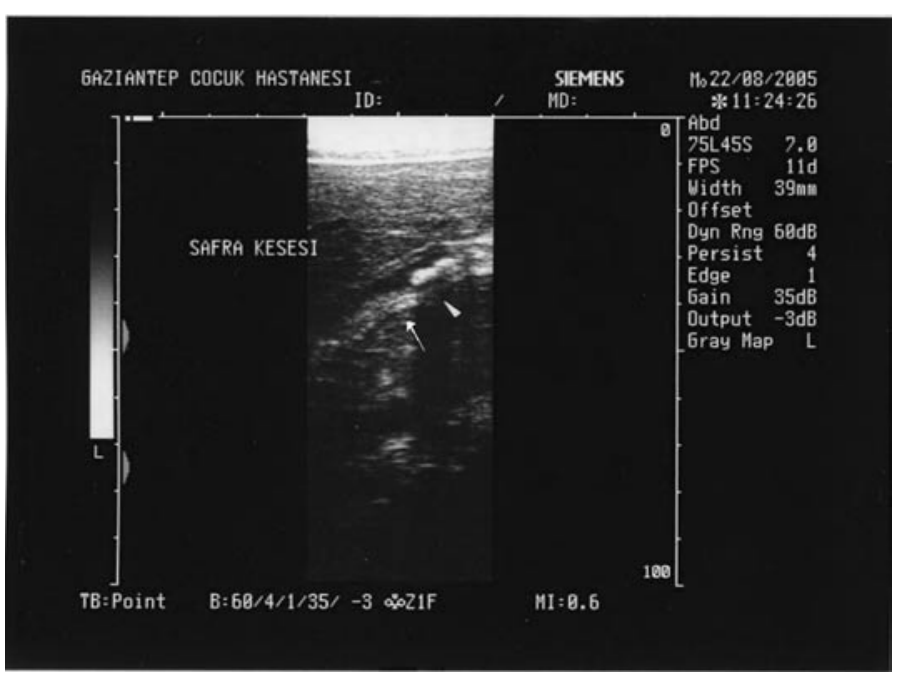

Fig. 3. Ultrasonographic image of biliary sludge with gallstone.

Arrowhead pointing to a gallstone with accompanying post-acustic shadows and arrow showing low-echogenic particles without post-acustic shadows indicating sludge in the gallbladder lumen.

TABLE 2. Time and results of the ultrasonographic examination.

\begin{tabular}{ccccc}
\hline Case & First US & Sludge & $\begin{array}{c}\text { Gallstone } \\
(\text { size })\end{array}$ & Follow-up US $^{\text {b }}$ \\
\hline 1 & 10th day & - & $7-8 \mathrm{~mm}$ & 60th day \\
2 & 7th day & - & Milimetric & 30th day \\
3 & 8th day & + & $5 \mathrm{~mm}$ & 30th day \\
4 & 8th day & + & - & 30th day \\
5 & 7th day & + & Milimetric & 90th day \\
6 & 7th day & + & Milimetric & 35th day \\
7 & 5th day & + & Milimetric & 20th day \\
8 & 6th day & - & $1.5 \mathrm{~cm}$ & 30th day \\
\hline
\end{tabular}

US, ultrasonography.

aFirst US was performed at $7 \pm 1$ days of the ceftriaxone treatment.

${ }^{\text {b}}$ Follow-up US was performed $41+23$ days after discontinuation of ceftriaxone.

All examinations were performed according to the institutional guidelines. All the parents had information about the study and oral informed consent was taken from all of them.

\section{Discussion}

Concentration of ceftriaxone in the gallbladder can exceed serum concentrations about 20 to 150 fold (Park et al. 1991). Because bile acids and ceftriaxone might use the same transport way, biliary secretion of ceftriaxone might inhibit the secretion of the bile acids. The concentration of ionized calcium in the bile is elevated. Ceftriaxone can precipitate with calcium like bilirubin. Thus, ceftriaxone-associated biliary sludge is composed mainly of calcium-ceftriaxone complexes (Park et al. 1991; Kim et al. 1992). Furthermore, in a recent experimental study by Arpacik et al. (2004) it was proposed that ceftriaxone could affect the gallbladder contractility.

Ceftriaxone-associated biliary sludge was first reported by Schaad et al. (1986). Biliary 
sludge has been usually accepted as a benign condition and clinical signs are usually absent. Symptoms like right upper quadrant pain, nausea, vomiting and cholecystitis occur only in a minority of the patients (Schaad et al. 1988; Schiffman et al. 1990; Papadopoulou et al. 1999). All subjects were asymptomatic in the study group and showed no biochemical features of cholestasis. Gallstone pancreatitis and choledocholithiasis are rare complications of ceftriaxone therapy reported previously (Robertson et al. 1996; Maranan et al. 1998).

Ceftriaxone therapy has been reported to be related more with biliary sludge rather than gallstones. However, only 2 of 8 patients of our study group had biliary sludge alone. The remaining 6 patients had gallstones with or without biliary sludge, but most of the gallstones were less than 3 $\mathrm{mm}$ in diameter.

Biliary precipitations form after approximately 9 days of treatment and usually resolve completely within 2-63 days after discontinuation of ceftriaxone therapy (Schaad et al. 1988). The formation of biliary sludge is also reported within the first 3 days of the treatment in more than half of the patients receiving ceftriaxone (Papadopoulou et al. 1999). High daily dosages and long-term duration of ceftriaxone therapy seems to be the main risk factors for the development of biliary sludge. Ceftriaxone dose was 100 $\mathrm{mg} / \mathrm{kg} /$ day in our study and mean duration of the treatment was $7 \pm 1$ days.

The incidence of ceftriaxone-associated biliary sludge was reported between 17-57.5\% (Schaad et al. 1988; Papadopoulou et al. 1999; Palanduz et al. 2000; Herek et al. 2001; Bor et al. 2004; Ozturk et al. 2005). Higher rates were reported with higher doses of ceftriaxone in those studies (100 mg/kg/day vs $60-70 \mathrm{mg} / \mathrm{kg} /$ day). The highest incidence for ceftriaxone-associated biliary sludge was reported by Ozturk et al. (2005). The authors explained the high incidence with several reasons: high dose of ceftriaxone (100 $\mathrm{mg} / \mathrm{kg} /$ day), restriction of oral intake in half of the patients and the high weather temperature in summer in their region. Especially the high temperature may be an important risk factor for pseudolithiasis. This may cause extreme loss of fluid, leading to easier formation of sludge. In the present study, 6 of the cases were diagnosed during the summer time. High weather temperatures exceeding $35^{\circ} \mathrm{C}$ is common in our region during the summer time. The probable effect of high temperature on biliary sludge formation is only an observation. The major limitation for the suggestion is that the study was not designed as a prospective study with a winter control group. High fever and restriction of oral intake of the study subjects could have resulted in dehydratation as well. Fasting and dehydratation are known risk factors for gallbladder dysfunction (Schiffman et al. 1990; Kong and Chen 1996).

In the present study group, some of the subjects were receiving additional antimicrobial agents. Although none of these drugs are known causes of biliary sludge, it may be speculated that these agents may facilitate the formation of sludge with ceftriaxone treatment.

In conclusion, the results of this study showed that biliary sludge may occur within the early days of ceftriaxone therapy. Pediatric patients receiving ceftriaxone treatment higher than $100 \mathrm{mg} / \mathrm{kg} /$ day in areas with high weather temperature have an increased risk for biliary sludge or gallstone formation. Therefore, clinicians should screen such patients by gallbladder ultrasonography during the ceftriaxone treatment even they are asymptomatic.

\section{References}

Arpacik, M., Ceran, C., Kaya, T., Karadag, B., Sarac, B. \& Koyluoglu, G. (2004) Effects of ceftriaxone sodium on in vitro gallbladder contractility in guinea pigs. J. Surg. Res., 122, 157-161.

Bor, O., Dinleyici, E.C., Kebapci, M. \& Aydogdu, S.D. (2004) Ceftriaxone-associated biliary sludge and pseudocholelithiasis during childhood: a prospective study. Pediatr. Int., 46, 322-324.

Herek, O., Pakdemirli, E. \& Kocer, N. (2001) Ceftriaxoneassociated biliary pseudolithiasis in children. Eur. Radiol., 11, 902.

Kim, Y.S., Kestell, M.F. \& Lee, S.P. (1992) Gallbladder sludge: lessons from ceftriaxone. J. Gastroenterol. Hepatol., 7, 618-621.

Ko, C.W., Sekijima, J.H. \& Lee, S.P. (1999) Biliary sludge. Ann. Intern. Med., 130, 301-311.

Kong, M.S. \& Chen, C.Y. (1996) Risk factors leading to ceftriaxone associated biliary pseudolithiasis in children. Chang Gung Med.J., 19, 50-54. 
Lopez, A.J., O’keefe, P., Morrisey, M. \& Pickleman, J. (1991) Ceftriaxone induced cholelithiasis. Ann. Intern. Med., 115, 712-714.

Maranan, M.C., Gerber, S.I. \& Miller, G.G. (1998) Gallstone pancreatitis caused by ceftriaxone. Pediatr. Infect. Dis. J., 17, 662-663.

Ozturk, A., Kaya, M., Zeyrek, D., Ozturk, N., Kat, N. \& Ziylan, S.Z. (2005) Ultrasonographic findings in ceftriaxone: associated biliary sludge and pseudolithiasis in children. Acta Radiol., 46, 112-116.

Palanduz, A., Yalcin, I., Tonguc, E., Guler, N., Ones, U., Salman, N. \& Somer, A. (2000) Sonographic examination of ceftriaxone-associated biliary pseudolithiasis in children. J. Clin. Ultrasound, 28, 166-168.

Papadopoulou, F., Efremidis, S., Karyda, S., Badouraki, M., Karatza, E., Panteliadis, C. \& Malaka, K. (1999) Incidence of ceftriaxone-associated gallbladder pseudolithiasis. Acta Pediatr., 88, 1352-1355.
Park, H.Z., Lee, S.P. \& Schy, A.L. (1991) Ceftriaxone-associated gallbladder sludge. Identification of calcium-ceftriaxone salt as a major component of gallbladder precipitate. Gastroenterology, 100, 1665-1670.

Robertson, F.M., Crombleholme, T.M., Barlow, S.E., Verhave, M. \& Brown, D. (1996) Ceftriaxone choledocholithiasis. Pediatrics, 98, 133-135.

Schaad, U.B., Tschahaeppeler, H. \& Lentze, M.J. (1986) Transient formation of precipitations in the gallbladder associated with ceftriaxone therapy. Pediatr. Infect. Dis., 5, 708-710.

Schaad, U.B., Wedgwood-Krucko, J. \& Tschaeppler, H. (1988) Reversible ceftriaxone-associated biliary pseudolithiasis in children. Lancet, 2, 1411-1413.

Schiffman, M.L., Keith, F.B. \& Moore, E.W. (1990) Pathogenesis of ceftriaxone-associated biliary sludge: in vitro studies of calcium-ceftriaxone binding and solubility. Gastroenterology, 99, 1722-1728. 